\title{
PAPER
}

\section{A model-based investigation of activations of the tongue muscles in vowel production}

\author{
Qiang Fang ${ }^{1, *}$, Satoru Fujita ${ }^{2}$, Xugang $\mathrm{Lu}^{3}$ and Jianwu Dang ${ }^{1}$ \\ ${ }^{1}$ Japan Advanced Institute of Science and Technology, \\ 1-1 Asahidai, Nomi, 923-1292 Japan \\ ${ }^{2}$ AI inc., \\ Tokuei-bldg. 8F, 5-33-7 Shiba, Minato-ku, Tokyo, 108-0014 Japan \\ ${ }^{3}$ ATR Spoken Language Communication Research Laboratories, \\ 2-2-2 Hikaridai, Keihanna Science City, Kyoto, 619-0288 Japan
}

(Received 16 June 2008, Accepted for publication 10 February 2009)

\begin{abstract}
Muscle activations during speech production are important for understanding speech motor control. In this paper, we put forward a physiological articulatory model-based approach to estimate muscle activations in producing five sustained Japanese vowels by minimizing the morphological difference between model simulations and target MRI observations, where the model is an improved version of Dang's partial 3D model. The initial muscle activations in the model simulation are set according to observation obtained by EMG measurement in producing vowels [6]. Then, the activation level of the tongue muscles are gradually adjusted so as to reduce the difference between the simulations and target MRI observations using an optimization approach. The results show that the proposed method can provide more details of the muscle activations than that obtained by EMG. In addition, the results suggest that the muscles Transversus and Verticalis play important roles in manipulating the length of tongue for vowel production; and, it is better to separate the Styloglossus into two control units, the anterior portion and posterior portion, in vowel production.
\end{abstract}

Keywords: Muscle activation, Speech production, Vowel production, Physiological articulatory model

PACS number: 43.70.Bk, 43.70.Jt [doi:10.1250/ast.30.277]

\section{INTRODUCTION}

During speech production, speech organs are driven by coordinated muscle activations to manipulate the vocal tract shape and provide proper sound source. Tongue is the most important speech organ that forms vocal tract shapes for producing most of the vowels and consonants. To understand how speech is controlled and how it is disrupted in various speech disorders, therefore, it is important to examine the activations of tongue muscles during speech production. The tongue is driven by activating a set of associate muscles when producing speech and other movements. Since most of those muscles locate deep inside the tongue body, it is difficult to measure the activations of the tongue muscles directly. For years, speech scientists have developed several methods to explore the activations of tongue muscles during speech production. Among them,

*e-mail: fangqiang@jaist.ac.jp
tagged-MRI [1-3] and Electromyography (EMG) [4-7] are the most popular tools.

The tagged-MRI is an approach that helps to trace the internal motion of a deformable body. Since the 1990's, it has been implemented to estimate activations of tongue muscles by comparing the measured length of muscle fibers [8-10] or principal strain of the tongue body $[1,11]$ with a reference configuration. Nonetheless, it has several inherent drawbacks. Firstly, it is difficult to define the reference configuration directly from the MR images and evaluate the rationality of the defined reference configuration. Secondly, since the tongue consists of volume preserving and interweaved muscles, the shortening of the muscle fibers may be caused either by their active contraction or by passive deformation in the associated area of the tongue, where only the active contraction of the muscles is what we are interested in. But these two kinds of shortenings can not be differentiated by using tagged-MR images alone. In addition, some of the muscles can be lengthened even if it 
is activated [6]. Nevertheless, this kind of muscle activation cannot be detected by tagged-MRI approach.

EMG is a technique that detects the electrical potential generated by muscle cells when the muscle is activated. Baer et al. [6] explored the activation of most of the major extrinsic muscles simultaneously, e.g., genioglossus posterior, genioglossus anterior, styloglossus, hyoglossus, geniohyiod, and mylohyiod, by inserting hooked wire electrodes into those muscles during producing English vowels in the context of /opvp/. Their results were widely referenced. However, it is difficult to measure the activations of small intrinsic muscles, such as transversus and verticalis, which may also play important roles in speech production, by using EMG.

To suppress problems of tagged-MRI and EMG in exploring muscle activations, we proposed a physiological articulatory model based method. It is assumed that if a faithful physiological articulatory model generates the same articulatory posture as the observed articulation, the muscle activations applied in the model are the possible ones that the speaker used in the observed articulation. In this way, the activations of major extrinsic muscles as well as small intrinsic muscles are possible to be estimated without suffering uncertain relation between the compression pattern of tongue and muscle activations.

In the literature, a number of physiological articulatory models have been proposed for various purpose, which were constructed in 2D [12-16], partial 3D [17,18], and 3D $[19,20]$. Although those models were used for the task of speech synthesis and investigating the motor commands, they have some deficiencies: (1) the 2D and partial 3D models only partially replicate of the tongue, thus, it cannot correctly represent the anatomical orientations of the tongue muscles; (2) the 2D and partial 3D models only partially replicate of the jaw and surrounding structures, thus, they cannot correctly account for the interactions between the tongue and surrounding structures; (3) the tongue structure, musculature, and surrounding vocal tract wall of the extant 3D model [19] are too coarse due to the trade off between anatomical accuracy and computational cost.

In this study, we firstly extend the previous partial 3D model [18] into a full 3D physiological articulatory model by combining a 3D tongue model [21] with the real jaw and vocal tract wall measured from MRI images, and use the model to investigate the muscle activations in the production of Japanese vowels.

\section{METHODS}

\subsection{D Physiological Articulatory Model}

In this study, we utilize a physiological articulatory model to estimate the activation of the tongue muscle by reproducing the articulatory observations for the Japanese

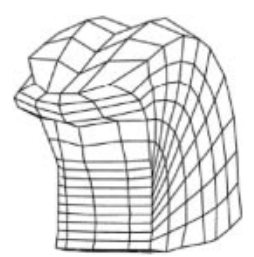

(a)

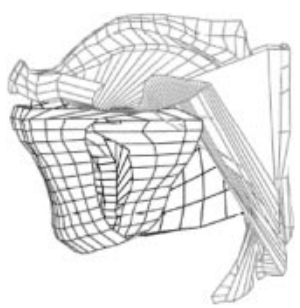

(b)
Fig. 1 (a) The oblique view of the 3D tongue involved in the 3D physiological articulatory model; (b) The profile of the $3 \mathrm{D}$ physiological articulatory model.

vowels. It requires the physiological articulatory model to be able to represent the human mechanisms faithfully in all of the anatomical, physiological and morphological aspects. To this end, we focus on realization of faithfulness of the model first. The proposed model is composed of the following components: the 3D tongue, jaw and vocal tract wall, and associated muscular and bony structures. In this part, we will introduce the details of the modeling.

\subsubsection{D tongue in the model}

The morphological structure of the 3D tongue is adopted from a computational tongue model for clinical application (see Fujita et al. [21] for details). The initial shape of the tongue is obtained based on the volumetric MR images while producing the Japanese vowel /e/. The mesh structure of the tongue is similar to the partial 3D model [18] except that, in the left-right dimension, the new tongue model adopts the realistic shape of the MRI-based tongue tissue geometry using five layers. Figure 1(a) demonstrates the configuration of the 3D tongue involved in the physiological articulatory model. The physical parameters for the 3D tongue model are the same as those in Fujita's model [21].

\subsubsection{Jaw and vocal tract wall in the model}

In this model, the contours of the jaw and vocal tract wall are carefully extracted form the MRI images of vowel /e/ superimposed with the lower and upper teeth [22] at the interval of $0.4 \mathrm{~cm}$ in the transverse dimension. Finally, the vocal-tract wall and the jaw consist of 15 layers, respectively, and the distance between the left and right extremes is $5.6 \mathrm{~cm}$. The left extreme layer is on the outer surface of the left side molar, and right extreme layer is on the outer surface of the right side molar. Accordingly, the vocal-tact wall and jaw can form a complete enclosed space when the jaw clenches with the maxilla rather than a partial vocal tract bounded by two parallel sagittal boundaries in the partial 3D model [18].

In the current model, the vocal-tract wall is composed of the upper teeth, hard palate, velum, pharyngeal wall, and larynx tube. The position of the velum can be adjusted 


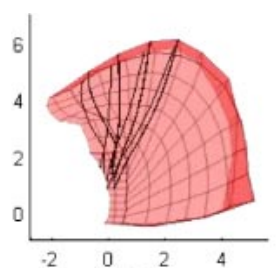

(a) GGa

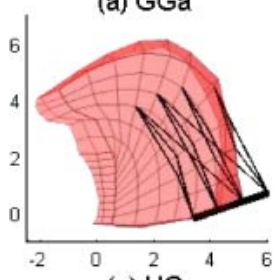

(e) HG

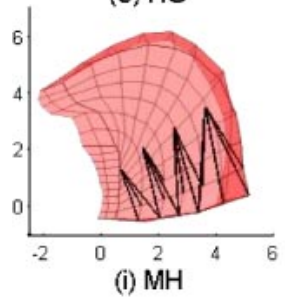

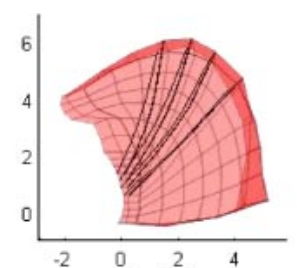

(b) GGm
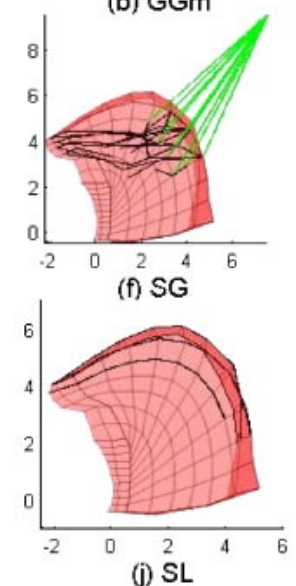

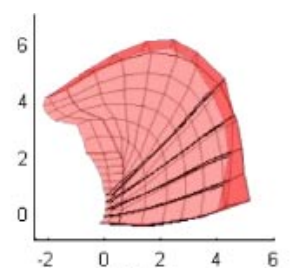

(c) GGp

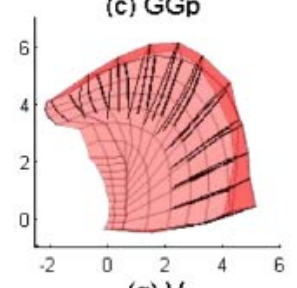

(g) $\mathrm{V}$

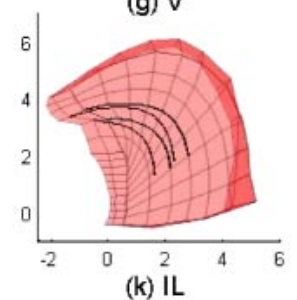

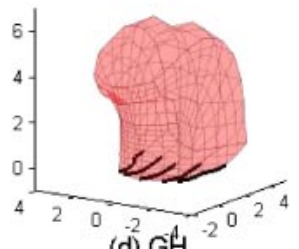

(d) $\mathrm{GH}$
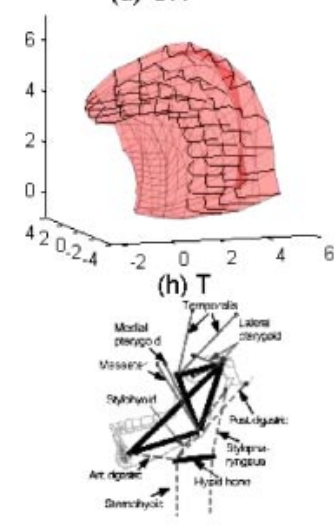

(1) Jaw-Hyoid Complex

Fig. 2 The musculature and jaw-hyoid complex of the 3D physiological articulatory model. (a) Genioglossus Anterior (GGa); (b) Genioglossus Middle (GGm); (c) Genioglossus Posterior (GGp); (d) Geniohyoid (GH); (e) Hyoglossus (HG); (f) Styloglossus (SG); (g) Verticalis (V); (h) Transversus (T); (i) Mylohyoid (MH); (j) Superior Longitudinal (SL); (k) Inferior Longitudinal (IL); (1) jaw-hyoid complex (solid segments: jaw-closing muscle group; dashed segments: jawopening muscle group; thick lines: rigid beams).

according to the size of velo-pharyngeal port, which is for generating nasal sounds. Other parts of the vocal tract wall are treated as rigid components. Figure 1(b) demonstrates the 3D physiological articulatory model.

The physical parameters for the jaw are the same as those in the partial 3D model [21].

\subsubsection{Musculature in the model}

To drive the physiological articulatory model according to human mechanism, the associated muscles in the model are arranged based on their anatomical orientations. There are nine muscles included in the tongue model. Three extrinsic muscles, genioglossus (GG), styloglossus (SG), and hyoglossus (HG), are arranged mainly based on the MRI analysis [17]. The intrinsic muscles, superior longitudinal (SL), inferior longitudinal (IL), transversus (T), verticalis $(\mathrm{V})$, are modeled based on the anatomical data from Takemoto [23]. The tongue floor muscles, mylohyoid $(\mathrm{MH})$ and geniohyoid (GH), are arranged based on anatomical literature [6]. All the muscles are arranged in bilateral symmetry. According to the function of the different parts of GG [4,5,7], GG is divided into three portions: GG anterior (GGa), GG middle (GGm), and GG posterior (GGp). SG is divided into 2 parts $\mathrm{SGa}$ (the part within the tongue) and SGp (the part from the styloid process to the insertion) to offer more degrees of freedom in manipulating the model. Figure 2(a)-(k) illustrates the layout of the major extrinsic and intrinsic muscles in the 3D physiological articulatory model.
Figure 2(1) shows the model of the jaw-hyoid complex, which are the same as that used in the partial 3D model [18], except the dimension in the transverse direction. The frame of the jaw is modeled by five rigid beams (the upper thick lines), and the hyoid bone is modeled as three segments corresponding to the body and bilateral greater horns (the lower thick lines). Although there is no unique mapping between muscle activations and kinematic degrees of freedom, the muscles involved in the jaw movements during speech can be roughly separated into two groups: the jaw-closer group (jawCl: solid segments) and the jaw-opener group (jawOp: dash segments).

\subsection{Exploring Muscle Activations in Vowel Produc- tion}

Since the proposed 3D model is more realistic in both morphological structure and musculature of the tongue and surrounding organs, the muscle activations during speech production are expected to be reliably estimated by using the proposed 3D model. For this reason, we estimate the muscle activation by using the proposed 3D physiological articulatory model via an optimization procedure, which minimizes the differences between model simulations and corresponding MRI observations.

\subsubsection{MRI based observation of vowel production}

In this study, we used the ATR MRI database as the observations of vowel production, which include the sagittal configuration of five Japanese vowels /a/, /i/, 
$/ \mathrm{u} /, / \mathrm{e} /$, and $/ \mathrm{o} /$. To depict deformation of the tongue in the transverse dimension, the tongue width are measured by using the distance between left and right landmarks (the sharp bending points of the deep branches of the lingual arteries, see Takano et al. [25] for details) in producing the five vowels.

\subsubsection{Cost function for optimization}

To increase the accuracy of the estimated muscle activation in vowel production, we proposed an optimization procedure to minimize the difference between model simulations and target articulations by automatically adjusting activation levels of the associated muscles. The cost function is defined to depict the difference between the simulated and observed postures in $3 \mathrm{D}$, as shown in Eq. (1). The right side of Eq. (1) is the summation of two terms: the first term accounts for the difference of the tongue contours in the midsagittal plane, and the second term is related to the difference in the transversal dimension. Consequently, this equation accounts for the $3 \mathrm{D}$ difference of the tongue shapes between the simulation and observation to some extent.

$$
D=\frac{\left(\boldsymbol{s}-\boldsymbol{s}_{0}\right)^{T}\left(\boldsymbol{s}-\boldsymbol{s}_{0}\right)}{N_{s}}+\frac{\left(\boldsymbol{w}-\boldsymbol{w}_{0}\right)^{T}\left(\boldsymbol{w}-\boldsymbol{w}_{0}\right)}{N_{w}}
$$

where $s_{0}$ and $s$ are the vectors that represent the midsagittal contours of the tongue in the observation and simulation, respectively; $\boldsymbol{w}_{0}$ and $\boldsymbol{w}$ are the tongue width vectors obtained from observation and simulation, respectively; $N_{s}$ and $N_{w}$ are the number of dimensions of vector $\boldsymbol{s}$ and $\boldsymbol{w}$, respectively. Both $s$ and $w$ are functions with regard to muscle activation $f$.

To calculate the difference between $s_{0}$ and $s$, we define a semi-polar coordinate system (as shown in Fig. 3), which avoids matching corresponding nodes between observation and simulation and approximately represents the crosssectional dimensions of the vocal tract. The contour of the tongue surface in the midsagittal plane is depicted by a sequence of lengths of the line segments that are nearly

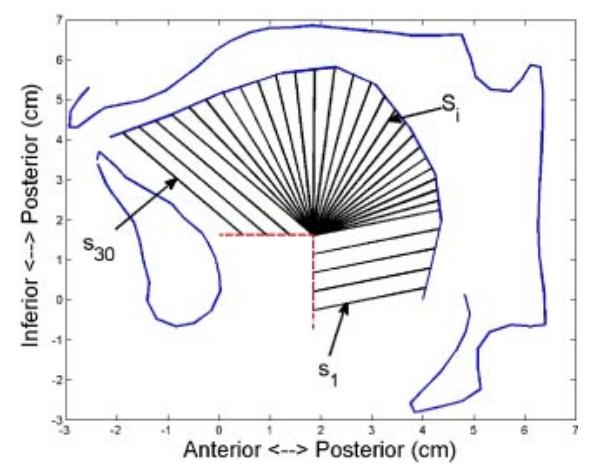

Fig. 3 The semi polar system for evaluating the difference between the real tongue shape and simulation result. perpendicular to the tongue surface $\left(s_{1}, s_{2}, \ldots, s_{30}\right)$, where $\mathrm{s}_{i}$ equals zero if tongue contour does not intersect with the $i$ th grid line. To depict the difference in the transversal dimension, the tongue width in the simulation is defined as the average distance between the 2 nd and 4th layers in the transverse dimension, which approximates the distance between the anatomical landmarks mentioned in Section 2.2.1. Since both $s$ and $w$ are the functions in terms of muscle activation $f$, estimating the muscle activation finally arrives at looking for the activation $f$ of the tongue muscles that are able to minimize the difference between observation and simulation. Nonetheless, it is difficult to define an analytic relationship between $\boldsymbol{s}$ and $\boldsymbol{f}$, and $\boldsymbol{w}$ and $f$ due to the complicated relationship between the tongue configuration and muscle activations. Hence, the gradient descent algorithm is implemented to estimate the underlying muscle activation, as defined in Eq. (2).

$$
f_{n}=f_{n-1}-\frac{0.05}{\lambda} \nabla_{f} D ; \lambda=\max \left\{\left|d_{i}\right| \mid i=1, \ldots, N_{f}\right\} ;
$$

where $f_{n}$ is the force vector at the current step, $f_{n-1}$ is the force vector at the previous step, $d_{i}$ is the ith element of vector $\nabla_{f} D$, and $N_{f}$ is the number of elements in vector $\nabla_{f} D$. To calculate the gradient of $D$ with regard to $f$, we adopt the method proposed by Shirai and Honda [30] to approximate the partial derivative vector. That is, for a given small variation of $\Delta f$ around $f_{n-1}$, we obtain the corresponding variation $\Delta D(f)$ by model simulation.

2.2.3. Procedure for estimating activations of tongue muscles

To make the simulated tongue shapes as close to the observations as possible and guarantee the rationality of the estimated activation patterns of tongue muscles, in this study, we start our estimation with reference to the observed EMG signals. Then, additional muscles or unnecessary muscles are activated or deactivated to reduce the difference between model simulations and objective observations based on the knowledge on the functions of tongue muscles. Comparing the anatomical orientations of the muscles in the proposed 3D model and the description on the position of the electrodes attached to in Baer's EMG experiment [6], we notice that the muscle GGa in Baer's experiment corresponds to the muscle GGm in our model, while the location of electrodes for HG, GGp, SG, MH and $\mathrm{GH}$ are consistent with the arrangements of the corresponding muscles implemented in the 3D model. Therefore, we can decide the initial setting with reference to their measurements. When inspecting the EMG observation presented by Baer et al., we noticed that the activation of muscle GGm ( $\mathrm{GGa}$ in Baer et al.'s experiments) in producing vowel $/ \mathrm{a} /, \mathrm{u} /$, and $/ \mathrm{o} /, \mathrm{SG}$ in producing vowel $/ \mathrm{i} /$, and $\mathrm{GH}$ in producing all the vowels were less than $1 / 8$ of the maximum observed muscle activation in the 


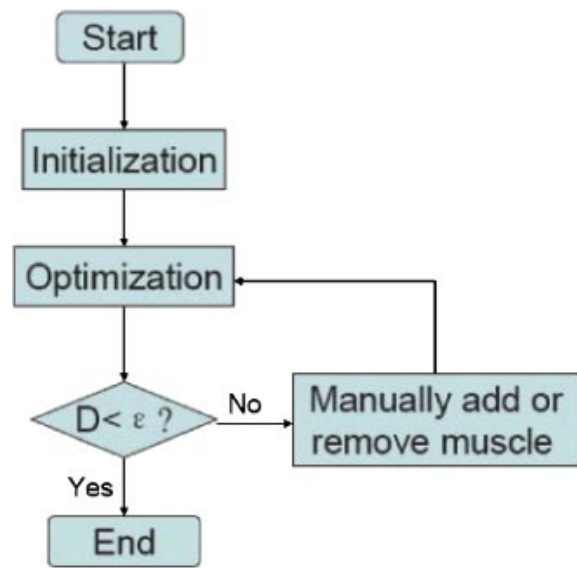

Fig. 4 The procedure for estimating the muscle activations during vowel production.

Table 1 The muscles used in the initial conditions (the second column) which are derived from the experiment of [6], and the additional muscles (the third column) recruited in the production of the vowels. The order of the muscle shows the order that the muscle is recruited in the procedure illustrated in Fig. 4.

\begin{tabular}{clc}
\hline Vowels & Muscle combinations & Additional muscles \\
\hline /a/ & SG, HG & T\&V, GGa \\
/i/ & GGm, GGp, MH & T\&V, GGa \\
/u/ & GGp, HG, SG & T\&V, GGa \\
/e/ & GGm, HG, MH, SG & GGa, T\&V \\
/o/ & SG, HG & T\&V, GGa \\
\hline
\end{tabular}

experiments. Accordingly, those muscles were treaded as inactive in the initial conditions.

Figure 4 shows the details of the procedure of the estimation of the muscle activation. At the step of initialization, firstly, the activation of $\mathrm{JawOp} / \mathrm{JawCl}$ is estimated by approximating the jaw in the model to the MRI observation. Then, the activated muscles observed in Baer's EMG experiments [6] are chosen as the initial muscle combinations (see Table 1 for details), and the initial activation levels are set according to relative amplitude of the EMG signals.

At step of optimization, the optimization method (see Section 2.2.2 for the details) is implemented to obtain the optimal muscle activation that minimizes the difference between simulation and target MRI observation with current muscle combination.

If the difference is greater than a predefined threshold, we refine the muscle combinations by manually activating additional muscle if necessary and/or deactivating the muscle with little contribution, then go back to the optimization step. If the difference reached the threshold, the optimization process terminates and the muscle activation patterns are obtained.

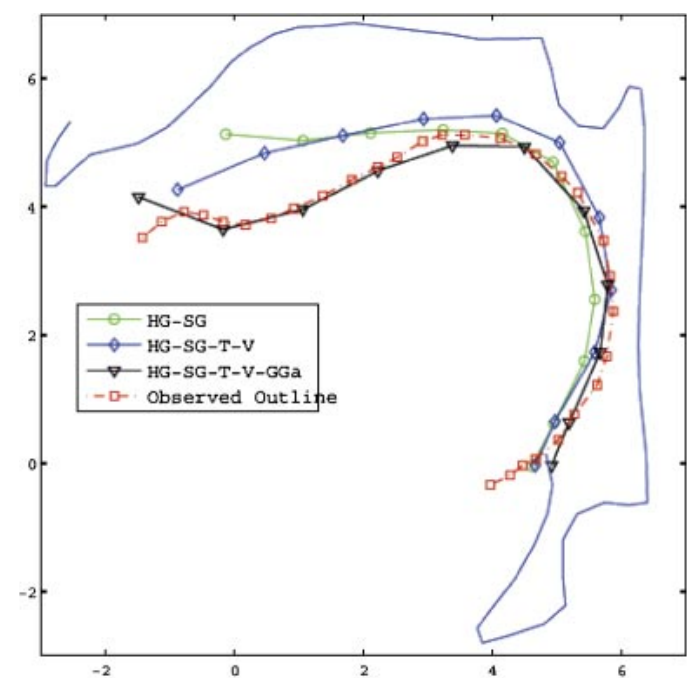

Fig. 5 An illustration of estimating activation of tongue muscles in producing vowel /a/. The curve consists of the squares is the observed outline of the tongue in the midsagittal plane. The curve consists of circles is the simulated outline of the tongue in the midsagittal plane by considering HG and SG only. The curve with diamonds is the outline the tongue by considering HG, $\mathrm{SG}, \mathrm{T}$ and $\mathrm{V}$. The curve with triangles is the outline of the tongue by considering $\mathrm{HG}, \mathrm{SG}, \mathrm{T}, \mathrm{V}$, and GGa.

\subsubsection{Illustration of the proposed method}

Figure 5 illustrates the estimation of the muscle activations in producing vowel /a/ by using the above procedure, where the observed tongue contour is indicated by the curve with square markers. When activating the muscles whose activation was observed in EMG experiment, we obtained the simulation shown by the curve with open circles. One can see that there is an obvious difference in the anterior portion although in the posterior portion the simulated contour of the tongue is consistent with the observation. Especially, the length of the contour of the simulation in the anterior portion is much shorter than that of the observation. Accordingly, to elongate the anterior part of the tongue, muscle $\mathrm{T}$ and $\mathrm{V}$ are selected by referencing the anatomical literature [29] and numerical experiments [31]. After considering the $\mathrm{T}$ and $\mathrm{V}$ in simulation, the tongue contour becomes closer to the observation, indicated by the curve with diamond makers. However, there are still differences between the simulation and the corresponding observation at tongue blade. This suggests that additional muscles are needed to depress this part. It is known that the GGa is an appropriate candidate with reference to the anatomical structure [23] and experimental data [1,9,32]. After taking GGa into account, the outline of simulation with inverse triangles becomes coincide with the observation. 


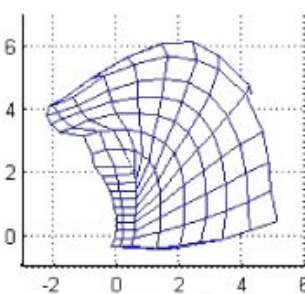

(a) rest

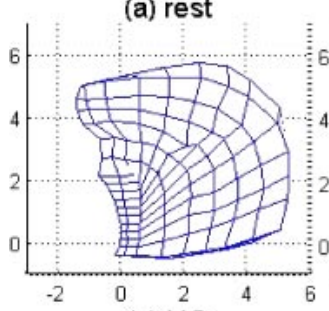

(e) HG

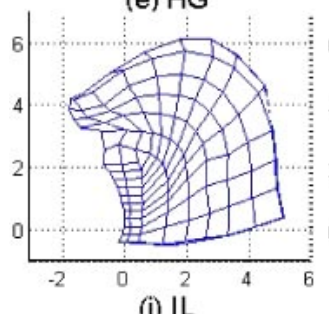

(i) IL

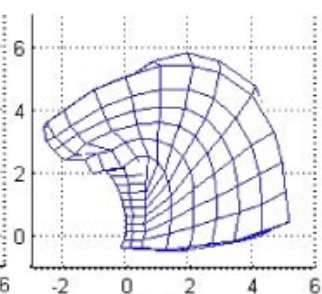

(b) GGa

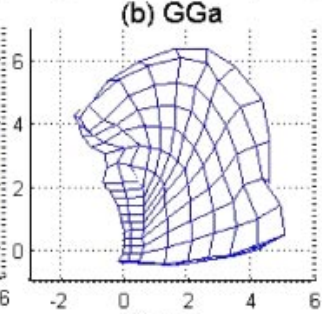

(f) $\mathrm{SGa}$

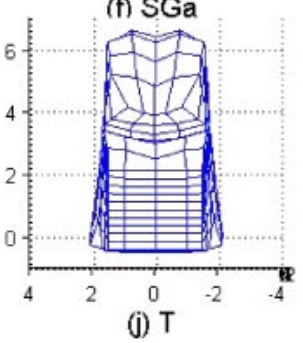

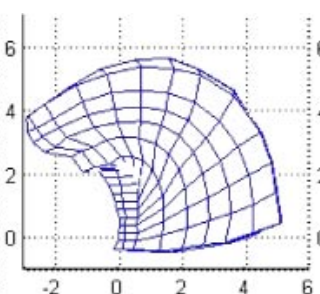

(c) GGm

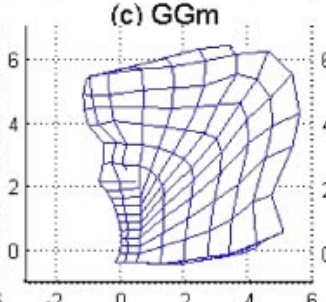

(a) SGp

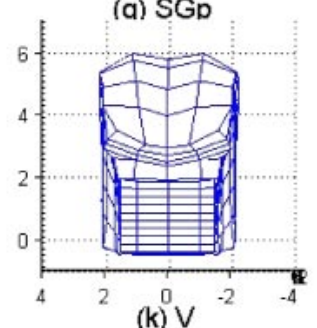

(k)

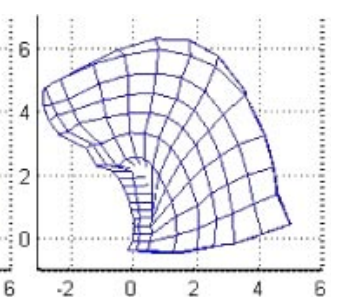

(d) GGp
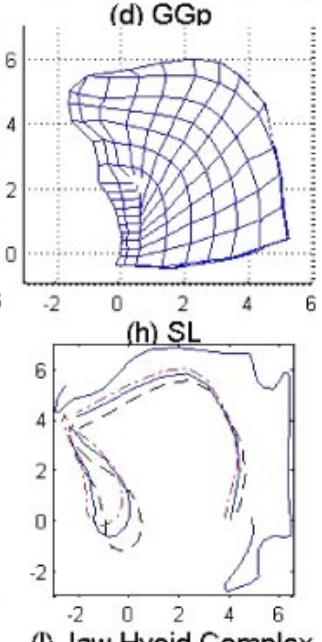

(I) Jaw-Hyoid Complex

Fig. 6 Results of activating individual tongue muscles, JawOp, and JawCl. (a) The configuration of the tongue without muscle activation; (b) GGa activated with $1 \mathrm{~N}$ force; (c) GGm activated with $1 \mathrm{~N}$ force; (d) GGp activated with $1 \mathrm{~N}$ force; (e) HG activated with $1 \mathrm{~N}$ force; (f) SGa activated with $1 \mathrm{~N}$ force; (g) SGp activated with $1 \mathrm{~N}$ force; (h) SL activated with $1 \mathrm{~N}$ force; (i) IL activated with $1 \mathrm{~N}$ force; (j) $\mathrm{T}$ activated with $1 \mathrm{~N}$ force; (k) $\mathrm{V}$ activated with $1 \mathrm{~N}$ force; (1) The position of the jaw and the tongue contour in the midsagittal plane under the various activations of muscle $\mathrm{JawOp} / \mathrm{JawCl}$ (solid curve: JawOp $0 \mathrm{~N}$; dashed curve: JawOp $3 \mathrm{~N}$; and dash-dotted curve: JawCl $3 \mathrm{~N}$ ).

\section{RESULTS}

\subsection{Testing of the Model}

In the literature, the function of the extrinsic and intrinsic muscles were qualitatively speculated based on anatomical descriptions $[1,24,25]$. By referencing those results, we tested the behavior of the 3D physiological articulatory model when activating the tongue muscles individually with $1 \mathrm{~N}$ in $300 \mathrm{~ms}$, where the boundary constraints on the tongue, resulted from the jaw and vocal tract wall, are not taken into account. Figure 6(a) gives the posture of the tongue at its rest position. Figure 6(b) demonstrates the result by activating GGa. Comparing with initial shape, the portion of the tongue blade is depressed. The activation of GGm (Fig. 6(c)) moves the tongue body downward and forward. The activation of GGp (Fig. 6(d)) makes the tongue body forward and slightly upward. The activation of HG (Fig. 6(e)) draws the tongue body downward and backward, while the tongue tip goes up with the rotation of the tongue body. Muscle SGa (Fig. 6(f)) helps to bunch the tongue. Muscle SGp (Fig. 6(g)) draws the tongue backward and upward. In addition, muscle SL (Fig. 6(h)) retracts the tongue body and elevates the tongue tip. Muscle IL (Fig. 6(i)) retracts the tongue body and makes the tongue tip slightly downward. The contraction of $\mathrm{T}$ narrows the tongue and elevates the tongue surface, as shown in Fig. 6(j), while the contraction of $\mathrm{V}$ draws tongue surface to the floor of the mouth, and makes it flatter and wider, as shown in Fig. 6(k). These results are consistent with muscle function speculated based on their anatomical orientation.

Moreover, the function of jawCl and jawOp are also tested. It shows that the jaw can clench with maxilla when $3 \mathrm{~N}$ force is applied to jawCl, and form the opening about $2 \mathrm{~cm}$ between jaw and maxilla in the midsagittal plane when $3 \mathrm{~N}$ force is applied to jawOp. The results are consistent with the observations reported by Ostry et al. [26].

\subsection{Differences between the Simulated and Observed Tongue Shapes}

Figure 7 shows the difference between the simulated tongue shape and observed tongue shape in the midsagittal plane and transversal dimension for the five vowels $/ \mathrm{a} /$, $/ \mathrm{i} /, / \mathrm{u} /, / \mathrm{e} /$, and $/ \mathrm{o} /$. The final configuration in the midsagittal plane is displayed by solid curves for the simulations and dashed curves for the target MRI observations, respectively. One can see that the simulations coincide with the target MRI observations when the model is driven by the optimized muscle activations. The average 


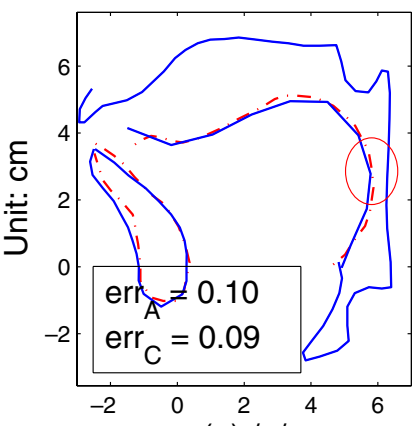

(a) /a/

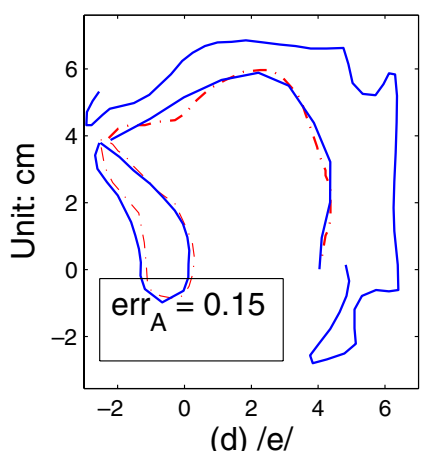

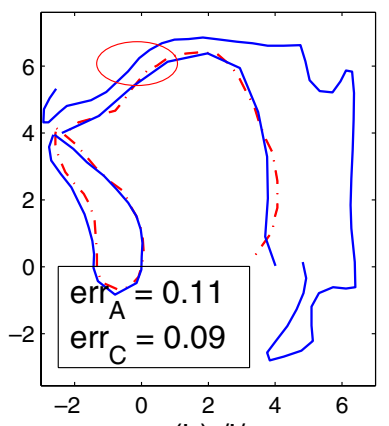

(b) /i/

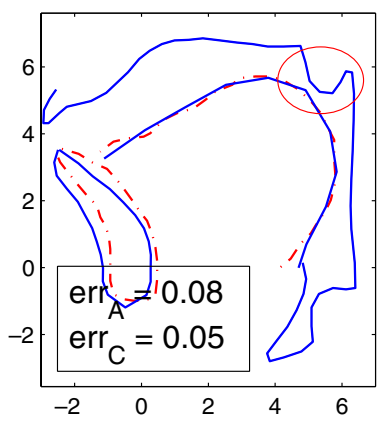

(e) /o/

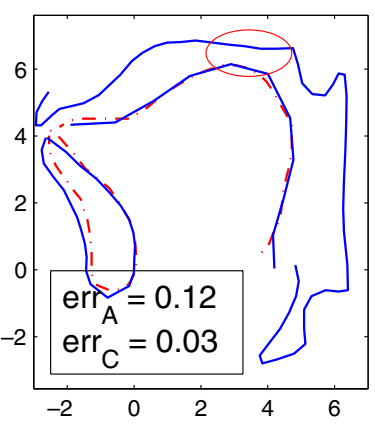

(c) $/ \mathrm{u} /$

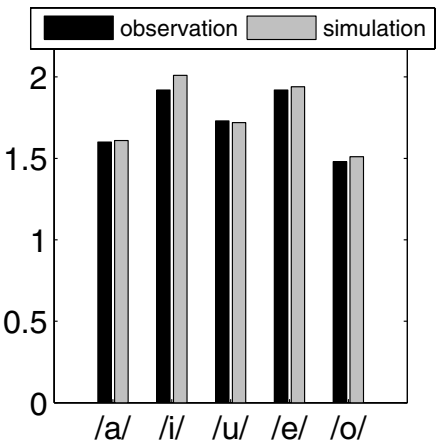

(f) width pattern of tongue

Fig. 7 The result obtained by proposed modeled based method. Panels (a-e) are the outline of tongue in the midsagittal plane for the observation (dashed curves) and simulation (solid curves) of vowel /a/, /e/, /u/, /e/ and /o/ respectively. Panel (f) is the relative tongue width pattern of observed (black bars) and simulated (gray bars) vowel /e/, /a/, /i/, /u/, and $/ \mathrm{o} /$ respectively.

errors (errA) of the simulations are $0.10,0.11,0.12,0.15$, and $0.08 \mathrm{~cm}$ for vowel $/ \mathrm{a} /, / \mathrm{i} /, / \mathrm{u} /, / \mathrm{e} /$ and $/ \mathrm{o} /$, respectively. The errors at the constrictions (errC), which are crucial for vowel production and indicated by ellipsis, are $0.09,0.09,0.03$, and $0.05 \mathrm{~cm}$ for $/ \mathrm{a} /, / \mathrm{i} /, / \mathrm{u} /$, and $/ \mathrm{o} /$ respectively. Figure 7(f) gives the tongue width changes in producing the five vowels $/ \mathrm{e} /, / \mathrm{a} /, / \mathrm{i} /, / \mathrm{u} /$, and $/ \mathrm{o} /$. A quantitative analysis indicates that the differences of the tongue width between the simulations and observations for each vowel are less than $0.07 \mathrm{~cm}$.

\subsection{Estimated Muscle Activations}

Figure 8 shows the muscle activations corresponding to the above simulated tongue shapes. The black bars are the estimated muscle activations in producing the five vowels by using the proposed method, and the gray bars are the corresponding normalized EMG observations. The muscle is activated in the model simulation if its activation level is non-zero in this figure. As a result, muscle GGa, HG, SGp, $\mathrm{T}$, and $\mathrm{V}$ are activated for producing vowel /a/; muscle GGa, GGm, GGp, SGa, SGp, T, V and MH are activated for producing vowel /i/; muscle GGa, GGp, HG, SGa, $\mathrm{SGp}, \mathrm{T}$ and $\mathrm{V}$ are activated for vowel /u/; muscle GGa, GGm, GGp, SGa, SGp, T, V, and MH are activated for vowel /e/, and muscle GGa, HG, SGa, SGp, T, V are activated for vowel /o/. It is found that muscles GGa, T, and $\mathrm{V}$ are active in the production of all the five vowels, although the levels of activation show some differences. In addition, the tongue muscles show relative stronger activation in producing four peripheral vowels $(/ \mathrm{a} /, / \mathrm{i} /$, $/ \mathrm{u} /$, and $/ \mathrm{o} /$ ) than that in producing central vowel $/ \mathrm{e} /$. HG and SGp show stronger activations in producing back vowels $/ \mathrm{a} /, / \mathrm{o} /$, and $/ \mathrm{u} /$ than in producing front vowel /i/ and $/ \mathrm{e} / \mathrm{GGa}$ and $\mathrm{MH}$ are activated with relative higher activation level for vowel /i/ and /e/ than for other vowels. GGp showed stronger activations for vowels /i/ and $/ \mathrm{u} /$ than for other vowels.

\section{DISCUSSIONS}

\subsection{Comparison of the 3D Model and the Partial 3D Model}

By extending the partial 3D model into full 3D model, both the morphological structure and the muscular arrangements of the model are improved. Accordingly, the 3D model has several advantages in exploring the mechanism of speech production.

4.1.1. Improvement of the morphological structure

The most important improvement is that the morphological structure of the tongue model completely replicates the real geometry of the tongue. In the partial 3D model, the tongue model represents the principal region of the tongue as a $2 \mathrm{~cm}$-thick layer bounded by three sagittal 


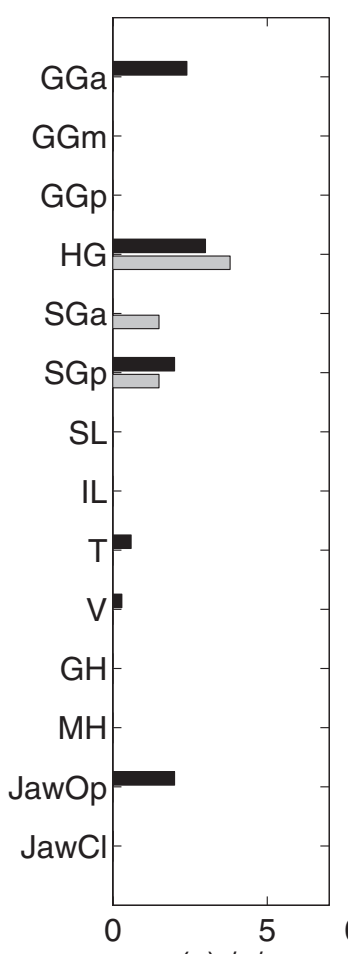

(a) /a/

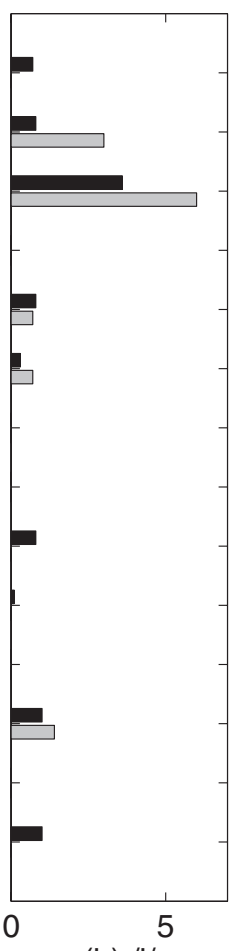

(b) /i/

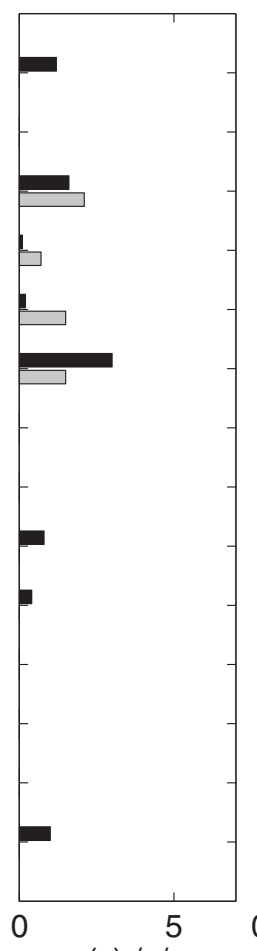

(c) $/ \mathrm{u} /$

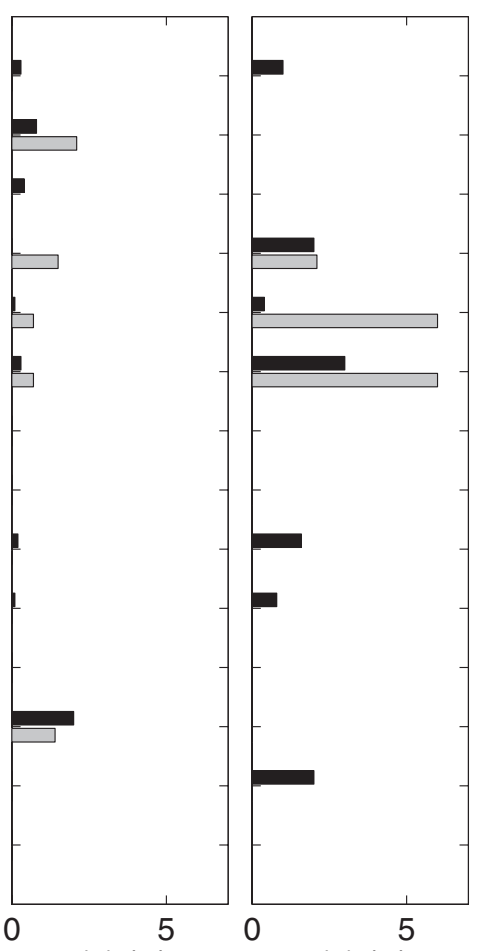

(d) /e/

(e) $/ 0 /$

Fig. 8 The activation of tongue muscles, in the production of five Japanese vowels, obtained by the 3D physiological articulatory model based method. The black bars are the results obtained by proposed method, and the gray bars are the corresponding normalized EMG measurements (Unit: Newton).

planes, which could not properly describe the tongue properties in the transverse dimension as well as the volume preserving properties of the tongue. In the current full 3D model, the tissue of the tongue in the left-right dimension is represented more realistically by extending the $2 \mathrm{~cm}$-think layer to real shape with a maximal width of $5.5 \mathrm{~cm}$ in the posterior part of the tongue. Consequently, the transverse dimension of the model is not only developed from three layers to five layers, but also the interval between the layers is changed with the tongue body dimension rather than the parallel arrangement. Therefore, the new tongue model is more realistic than the partial 3D model.

Companying with the improvements of the tongue model, the jaw and vocal tract wall are modeled according to the real structure based on volumetric MR images. As a result, the transversal dimension of the jaw and vocal tract wall are extended from $2.8 \mathrm{~cm}$ to $5.6 \mathrm{~cm}$.

Due to the above improvements, the interaction between tongue, jaw and vocal tract wall can be correctly modeled, and an enclosed vocal tract can be obtained during speech production. Thus, we can obtain more accurate cross-sectional area function of the vocal tract directly, but need not to estimate area function from the vocal tract width via the $\alpha-\beta$ model [27].

4.1.2. Improvement of the musculature

Another improvement of the proposed model is that orientations of the tongue muscles become more realistic. In the partial 3D tongue model, the arrangements of all the muscles were limited in three parallel layers, which is quite different from the real situation of the muscular structure. In the proposed 3D model, the muscle orientation is arranged as faithfully as possible based on the anatomical knowledge. Accordingly, muscle IL is arranged on the lateral sides of GG in different layers, instead of the previous arrangement in which they were located in the same layer. For the same reason, muscle HG is symmetrically located in the outside parasagittal planes. The anterior parts of SG is located on the outer lateral layers of GG, and the attach points of the SG to the stylo-process spans about $7.5 \mathrm{~cm}$ in transversal dimension, close to that of the human anatomic structure. The orientation of muscle $\mathrm{T}$ and $\mathrm{V}$ are completely replicated in the 3D model with reference to Takemoto's work [23], which was not able to be realized in the partial 3D model. Altogether, compared with the description of the muscle structures in anatomical literature $[23,28,29]$, the arrangement of the muscles in the proposed $3 \mathrm{D}$ model is more realistic. The realistic muscular structure makes it possible to obtain reliable estimation of muscle activations in speech production.

\subsubsection{Control unit of SG}

The muscle SG is known to play an important role in producing back vowels $/ \mathrm{u} /$ and $/ \mathrm{o} /$ [6]. However, there is little information on the control unit of muscle SG in 
speech production. Most of the physiological articulatory models $[12,14,15,18]$ treat the whole SG as one control unit. Recently, Takano et al. [25] found that the three parts of SG (SGa, SGm, and SGp see [25] for the details) showed different behaviors in producing Japanese vowels. The different behaviors may be resulted from separate controls on the different parts of SG.

With reference to Takano et al. [25], we divide the SG into two parts, the SGa (inside the tongue) and the SGp (from the styloid process to the insertion) in our 3D model to increase the degrees of freedom in model control. SGa and SGp are controlled independently. The results of muscle activations in vowel production demonstrated that the ratio of the activation of SGa to SGp (SGa/SGp) varies between 0 and 2.7 among the production of the five Japanese vowels, rather than always the same amount of ratio. This implies that, in vowel production, the SG should be separated, at least, into the anterior and posterior parts, and controlled independently.

\subsection{The Activations of Tongue Muscles in Vowel Production}

Kakita et al. [19] estimated the activation of tongue muscles with an simplified 3D physiological articulatory model, which is governed by quasi-static equations. However, in their study, they did not give the evaluation how similar are the simulated tongue shapes to real articulations, which is one of the important factors to evaluate the reliability of the estimated muscle activation. So, it is difficult to compare our results with their results directly.

Baer et al. [6] measured the activations of major extrinsic tongue muscles when producing /əpvp/ utterances. They found that: a) GGa, which is corresponding to GGm in our 3D model according to their description, and $\mathrm{MH}$ were more active for front vowel /i/ and /e/ than for back vowels; b) GGp was the most active in producing high vowel /i/ and /u/; c) HG and SG showed stronger activations in producing back vowels $/ \mathrm{a} /, / \mathrm{o} /$, and $/ \mathrm{u} /$. The results obtained by the proposed 3D model-based method are consistent with those observations. This suggests that the proposed method is able to discover reasonable muscle activation combinations in producing the five Japanese vowels.

\subsection{Function of GGa}

In this study, the GGa is defined as a subdivision of the GG that runs vertically in the anterior part of the tongue. The function of GGa has been widely investigated in vowel production. Stone et al. [1], and Niimi et al. [9] found that the anterior part of the tongue was vertically compressed in producing vowel /a/, and they associated this deformation pattern of the tongue with the activation of GGa. Takano et al. [25] found that GGa was shorter in back vowels than in front vowels. They suggested that GGa was activated in producing /a/ and /o/. In our study, we found that GGa was activated in producing all the vowels. It is widely accepted that the tongue is pushed forward and upward by activating the muscle GGp in producing vowel /i/ $[15,25,33,34]$, and the constriction formed by the anterior portion of the tongue and the corresponding part of the palate should be precisely controlled [35-37]. In our simulation, when we activated the muscles for producing the vowel /i/ as shown in Fig. 7, while deactivating GGa, the anterior portion of the tongue collapsed fully on the palate, and no airway is formed in this portion. Activating GGa forms a small duct in the anterior part by pulling down the midsagittal area of the tongue. This simulation supports the speculation about the function of GGa in producing high vowel $/ \mathrm{i} /$. These results are consistent with those found by Kakita et al. [19] in their model-based work.

\subsection{Function of $\mathbf{T}$ and $\mathbf{V}$}

So far, most previous studies focused on the function of the extrinsic muscles in vowel production. Only few of them tried to shed light on the functions of the intrinsic muscles $\mathrm{T}$ and $\mathrm{V}$ in vowel production. Takano et al. [33] found that $\mathrm{T}$ played an important role in elevating the tongue blade by comparing motion patterns obtained from tagged-MRI observations and the simulation based on a four-block tongue model. Stone et al. [1] claimed that the $\mathrm{V}$ contributed to the backing and lowering of the tongue surface in producing vowel /a/. However, nobody mentioned how the co-contraction of muscles $\mathrm{T}$ and $\mathrm{V}$ functions in vowel production. In this study, we found that the length of the tongue in MRI observation were $12.07,12.06,11.64,12.37,11.65$, and $12.90 \mathrm{~cm}$ for vowel /a/, /i/, /u/, /e/, and /o/, respectively. According to the anatomical structure of the tongue muscles, no muscle is directly responsible for elongating the tongue in longitudinal direction. Among the tongue muscles, the cocontraction of muscles $\mathrm{T}$ and $\mathrm{V}$ shrinks the cross-sectional area of the tongue and extend the tongue in the longitudinal direction due to the incompressibility of the soft tissue [21]. After taking the co-contraction of $\mathrm{T}$ and $\mathrm{V}$ into account, the tongue length are $11.85,12.23,12.46,11.73$, and 12.48 for vowel /a/, /i/, /u/, /e/, and /o/, respectively, which approximates the range of the tongue length variation in MRI observations. Meanwhile, the variations of the tongue width are consistent with the MRI observation, which is concerned with the activation of muscle $\mathrm{T}$. The consistency in $3 \mathrm{D}$ indicates that the cocontraction of intrinsic muscles $\mathrm{T}$ and $\mathrm{V}$ plays an important role in vowel production by controlling the length of the tongue. 


\section{CONCLUSION}

In this study, we put forward a physiological articulatory model-based approach to estimate the muscle activation, where the model is a version up of the partial 3D model [18], and evaluate the proposed method by numerical experiments. The estimation started from initializing the model based procedure with the normalized EMG signals [6], then estimated the optimal muscle activation patterns by minimizing the difference between the model simulation and MRI observation, where additional muscles were manually activated or deactivate according to their contribution to decreasing the difference. In this process, the extrinsic muscles that were observed in EMG experiments [6] are robustly appeared in the estimation. This verified that our method is capable to estimate the muscle activations. Moreover, the model based estimation method revealed that muscle $\mathrm{T}, \mathrm{V}$, and GGa are activated in producing all the five Japanese vowels. $\mathrm{T}$ and $\mathrm{V}$ play an important role in vowel production by manipulating the length of the tongue contour. GGa shows important function in active control of tongue blade.

In order to provide more flexibility to the proposed 3D model, we separated the SG into two portions for the control of the model: anterior portion (inside the tongue), and posterior portion (from the styloid process to the insertion). The estimated results of muscle activations indicate that the two-part treatment of SG is appropriate and necessary to derive optimal matching between the model simulations and the MRI observations.

In this study, we used a relative simple task, vowel production, to testify our proposed estimation method. The results implied that the $3 \mathrm{D}$ physiological articulatory model based estimation method is available and powerful to investigate the muscle activations of the intrinsic muscles as well as the extrinsic muscles in speech production.

\section{ACKNOWLEDGEMENT}

This research is conducted as a program for the " 21 st Century COE Program" for promoting Science and Technology by Ministry of Education, Culture, Sports, Science and Technology. This study is also supported in part by SCOPE (071705001) of Ministry of Internal Affairs and Communications (MIC), Japan.

\section{REFERENCES}

[1] M. Stone, "Modeling the motion of the internal tongue from tagged cine-MRI images," J. Acoust. Soc. Am., 109, 29742982 (2001).

[2] S. Kiritani, K. Miyawaki, O. Fujimura and J. E. Miller, "A computational model of the tongue," Ann. Bull. RILP, 10, 243251 (1976).

[3] K. Hashimoto and K. Sasaki, "On the relationship between the shape and position of the tongue for vowels," J. Phonet., 10, 291-299 (1982).

[4] P. MacNeilage and G. Sholes, "An electromyographic study of the tongue during vowel production," J. Speech Hear. Res., 7, 209-232 (1964).

[5] T. Smith, "A phonetic study of the functions of the extrinsic tongue muscles," UCLA Work. Pap. Phonet., No. 18 (1971).

[6] T. Baer, J. Alfonso and K. Honda, "Electromyography of the tongue muscle during vowels in /əpvp/ environment," Ann. Bull. RILP, 7, 7-18 (1988).

[7] K. Miyawaki, "A prelimilary report on the electromyographic study of the activity of lingual muscles," Ann. Bull. RILP, 9, 91-106 (1975).

[8] M. Kumada, M. Niitsu, S. Niimi and H. Hirose, "A study on the inner structure of the tongue in the production of the 5 Japanese vowels by tagging snapshort MRI," Ann. Bull. RILP, 26, 1-13 (1992).

[9] S. Niimi, M. Kumada and M. Niitsu, "Functions of tongue related muscles during production of the five Japanese vowels," Ann. Bull. RILP, 28, 33-40 (1994).

[10] M. Niitsu, M. Kumada, S. Niimi and Y. Itai, "Tongue movement during phonation: A rapid quatitative visualization using tagging snapshot MRI imaging," Ann. Bull. RILP, 26, 149-156 (1992).

[11] J. Dang, S. Fujita, E. Murano and M. Stone, "Observation and simulation of Large-scale deformation of tongue," in ISSP06, Brazil (2006).

[12] J. S. Perkell, "A physiologically-oriented model of tongue activity in speech production," PhD. Thesis, MIT (1974).

[13] P. Perrier, H. Lavenbruck and Y. Payan, "Control of tongue movments in speech: The Equilibrium Point Hypohtesi perspective," J. Phonet., 24, 53-75 (1996).

[14] Y. Payan and P. Perrier, "Synthesis of V-V sequences with a 2D biomechanical tongue model controled by the equilibrium point hypothesis," Speech Commun., 22, 185-205 (1997).

[15] K. Honda, "Orgnization of tongue articulation for vowels," $J$. Phonet., 24, 39-52 (1996).

[16] V. Sanguineti, R. Laboissiere and D. Ostry, "A dymamic biomechanical model for neural control of speech production," J. Acoust. Soc. Am., 103, 1615-1627 (1998).

[17] J. Dang and K. Honda, "A physiological model of a dynamic vocal tract for speech production," Acoust. Sci. \& Tech., 22, 415-425 (2001).

[18] J. Dang and K. Honda, "Construction and control of a physiological articulatory model," J. Acoust. Soc. Am., 115, 853-870 (2004).

[19] Y. Kakita, O. Fujimura and K. Honda, "Compuation of mapping from muscular contraction patterns to formant patterns in vowel space," in Phonetic Linguistics, V. A. Fromkin, Ed. (Academic Press, New York, 1985), pp. 133144.

[20] R. Wilhelms-Tricarico, "Physiological modeling of speech production: Methods for modeling soft-tissue articulators," $J$. Acoust. Soc. Am., 97, 3085-3098 (1995).

[21] S. Fujita and J. Dang, "A computational tongue model and its clinical application,” Oral Sci. Int., 4, 97-109 (2007).

[22] H. Takemoto, K. Kitamura, H. Nishimoto and K. Honda, "A method of tooth superimposition on MRI data for accurate measurement of vocal tract shape and dimensions," Acoust. Sci. \& Tech., 25, 468-474 (2004).

[23] H. Takemoto, "Morphological analysis of the tongue musculature for three dimensional modeling," J. Speech Hear. Res., 44, 95-107 (2001).

[24] J. Warfel, The Head, Neck, and Trunk (Led \& Febiger, Philadelphia and London, 1993). 
[25] S. Takano and K. Honda, "An MRI analysis of the extrinsic tongue muslces during vowel production," Speech Commun., 49, 49-58 (2007).

[26] D. J. Ostry and K. G. Munhall, "Control of jaw orientation and position in mastication and speech," J. Neurophysiol., 71, 1528-1545 (1994).

[27] J. Dang and K. Honda, "Estimation of vocal tract shape from sounds via a physiological articulatory model," J. Phonet., 30, 511-532 (2002).

[28] K. Miyawaki, "A study on the muscularture of the human tongue," Ann. Bull. RILP, 8, 23-49 (1974).

[29] W. R. Zemlin, Speech and Hearing Science: Anatomy and Physiology, 4th ed. (Allyn \& Bacon, Boston, 1998).

[30] K. Shirai and M. Honda, "Estimation of articulatory parameters from speech sound," Trans. IEICE, 61, 409-416 (1978).

[31] Q. Fang, S. Fujita and J. Dang, "Investigation of functions of tongue muscles for model control," Chin. J. Phonet. (in press).

[32] E. Davis, A. Douglas and M. Stone, "A continuum mechanics representation of tongue motion in speech," in ICSLP1996, Philadelphia, USA (1996).

[33] S. Takano, H. Matsuzaki and K. Motoki, "Investigation of the intrinsic tongue muscles for production of /i/ using tagged cine-MRI and a four-cube FEM model," in Proc. 2nd Int. Symp. Biomechanics, Healthcare and Information Scienc, Kanazawa, Japan (2008).

[34] J. S. Perkell, "Properties of the tongue help to define vowel categories: Hypotheses based on physiological-orineted modeling," J. Phonet., 24, 3-22 (1996).

[35] G. Fant, Acoustic Theory of Speech Production (Moution \& Co., The Hague, 1960).

[36] K. N. Stevens, "The quantal nature of speech: Evidence frome articulaotry-acoustic data," in Human Communication: A Unified View (McGraw-Hill, New York, 1972).

[37] K. N. Stevens, "On the quantal nature of speech," J. Phonet., 17, 3-45 (1989). 